\title{
Emphysematous Pyelonephritis
}

\author{
Balagobi $B^{1}$, Nitharsanan $M^{1}$, Thanusan $V^{2}$
}

${ }^{1}$ Department of Surgery ,Faculty of Medicine Jaffna

${ }^{2}$ National Hospital, Colombo, Srilanka

\begin{abstract}
Emphysematous pyelonephritis(EPN) is a potentially fatal manifestation of necrotizing infection of renal parenchymal tissues and perirenal tissues with gas formation in renal system. Its clinical significance due to its diagnostic difficulty on clinical grounds, rapid progression and the demand for the invasive management strategies in short term necessitates the need for a more vigilant approach in vulnerable population. Risk population includes female diabetic patients, obstructed infected urinary system, immunosuppression. Since its initial reporting from 19th century the treatment strategies are more towards extensive invasive approaches due to its rapid progression into fatal condition. Fortunately, with the advent of modern imaging facilities, the diagnostic difficulties were somewhat alleviated which helps in staging the disease. Thence the treatment strategy evolved over time which includes various options in treatment regime. Currently the treatment for emphysematous pyelonephritis includes resuscitation with fluid electrolyte management, intravenous antibiotics to cover gram negative bacteria, risk factor management, minimally invasive approaches towards decompression and in worst case scenario immediate or delayed nephrectomy. Here, we discuss the clinical features, aetiopathogenesis, diagnostic approaches and the treatment strategies of this uncommon but potentially fatal condition.
\end{abstract}

\section{Keywords}

Emphysematous pyelonephritis, pyelonephritis, Diabetes mellitus, nephrectomy

\section{Introduction}

Emphysematous pyelonephritis (EPN) is an acute severe form of necrotizing infection of renal parenchyma and surrounding tissue that results in accumulation of gas in the renal parenchyma, collecting system and perinephric tissue (1). It is often life-threatening. Kelly and Macculum reported the first case of gas forming renal infection in 1898. Initially EPN was described as renal emphysema and pneumonephritis. Later, Schultz and Klorfein recommended the term 'Emphysematous Pyelonephritis' (EPN) in 1962.

Its occurrence is mostly associated with the presence of diabetes mellitus(More than $80 \%$ of patients with EPN are diabetes) with a female preponderance (6times commoner than men) $(2,3,4,5)$ as well. Elderly population is more vulnerable due to their co existing other risk factors and co morbidities.

Other contributing factors are renal failure, immunosuppression, obstructed upper urinary tract and polycystic kidney disease $(5,6)$.EPN is typically characterized by fever and flank pain with radiological evidence of gas accumulation within and around the kidney. Even though the presentation is similar to acute pyelonephritis, EPN is more fatal if not recognized and treated properly and promptly.

Earlier, emergency nephrectomy and/or open surgical drainage with antibiotic coverage was the preferred management of patients with EPN. However, with the evolution of medical advances, a nephron-sparing approach using percutaneous catheter drainage(PCD) became a popular option for majority of the cases with better success rate. Yet some patients require subsequent nephrectomy as a part of life saving intervention in severe cases.

In order to make decision making more convenient various prognostic classification system exist.

Based on features observed on a CT scan, EPN can be classified into four which might explain the extent of the disease and about possible prognostic status(4). 
Class 1: Gas in the collecting system only.

Class 2: Gas in the renal parenchyma without extension to external space.

Class 3: 3A-Extension of gas or abscess to perinephric space. 3B- Extension of gas beyond the Gerota fascia.

Class 4: Bilateral EPN or Solitary kidney with EPN.

There is another method of prognostic classification of EPN from the CT scan appearance.

Type 1-Gas accumulated in the renal parenchymain a streaky/mottled pattern with evidence of necrosis.

Type 2- Gas accumulated in the collecting system of the kidney in a bubbly or loculated pattern.

According to this classification, patients with type1 EPN has more fulminant course of illness which might get worsen rapidly and high mortality rate than patients with type 2 EPN. It is because of the associated thrombosis and renal parenchymal necrosis in the type 1 .

Among these two classification systems, former categorization is more convenient as it not only provides an anatomical extent of the disease but also guides about the management approach according to the type.

\section{Aetiology and pathogenesis}

The mean age of a patient with EPN is 55 years. Females are having 6 times more chance to get this condition than men. It may be partly due to their increased vulnerability to urinary tract infections. Likewise, left kidney is more susceptible than right kidney due to its high likelihood of getting obstructed (7). Diabetes mellitus and immunosuppression are the major risk factors for the condition. Renal failure, obstructed upper urinary tractand polycystic kidney disease are the other contributing factors to the $\operatorname{EPN}(5,6)$. In non-diabetic patients major associated pathology is urinary tract obstruction which is mostly due to the papillary necrosis (5) and to a smaller extent by the ureteral calculi also.

EPN is commonly caused by enteric Gram-negative facultative anaerobes with Escherichia coli being the commonest organism(66\%) isolated in cultures. Klebsiella(26\%), Proteus,Pseudomonas, Streptococcus, enterococcus and clostridium species are other causative organisms. Mixed infection is found in about $10 \%$ of patients $(4,8,9)$. Organisms like Aspergillus fumigatus, Candida species and Entamoeba histolytica can also cause EPN but are of rare incidence.

The pathogenesis ofEPN is not clearly understood. Elevated blood and tissue glucose levels have been observed in diabetic patients. This may contribute to the formation of a favourable environment for gas forming bacteria that ferment glucose substrate at tissue level, resulting in carbon dioxide production (10). Formed gas is then entrapped due to any obstructive pathology which might coexist (11). Though this theory has been proposed as the cause for gas formation in the renal tissue, analysis of the gas content in EPN demonstrated nitrogen as the major component (60\%). Another theory suggests the association of severe necrotizing infections in renal parenchyma which leads to the above condition (11).

Around $15 \%$ of the gas was hydrogen and $5 \%$ was carbon dioxide. Diabetic angiopathy has also been proposed to contribute to the accumulation of gas by slowing the transport of catabolic products.

Urinary tract obstruction is also another major risk factor that can precipitate this fatal condition(4) which necessitates the urgent decompression as one of the immediate and lifesaving interventions in such patients. This kind of obstruction can be caused by mainly papillary necrosis. Ureteric calculi also contribute to a smaller proportion in such patients.

\section{Clinical presentation}

Clinical features are moreover similar to severe acute pyelonephritis and often indistinguishable.

The onset of symptoms can be abrupt or gradual over 2 to 3 weeks. The typical presentation of EPN is fever, abdominal or flank pain, nausea, vomiting and acute renal failure. In worst case scenario the disease takes a fulminant course where patients may present with shock, disturbance of consciousness, visible hematuria, severe proteinuria and features 
of disseminated intravascular coagulation such as thrombocytopenia and coagulopathy. Thus, early diagnosis is cardinal in managing these patients and it is advisable to keep a low index of suspicion in patients with high risk profile.

Pneumaturia is uncommon, but it can be observed in patients with associated emphysematous cystitis. $(2,8)$ Crepitus may be felt over the flank area in advanced cases. They usually fail to respond to IV antibiotics alone within 2-3 days.

Other differential diagnosis includes Xanthogranulo matous pyelonephritis and papillary necrosis with similar clinical presentations.

The presence of air either in or adjacent to the renal parenchyma also seen in renal abscess, entero-renal and cutaneo-renal fistula or it can be a reflux of air from urinary bladder following urological intervention. Distinction should be made upon clinical grounds as early as possible in these conditions as any delay in timely intervention in EPN would lead to devastating complications.

\section{Diagnosis}

Early diagnosis and treatment are mandatory in EPN as it's a serious life-threatening condition.In addition to the clinical presentation the diagnosis is usually made by radiological imaging of abdomen. Confirmation of the diagnosis is aided by the presence of intra renal gas shadow in computed tomography $(\mathrm{CT})$ which is more sensitive than plain films and may show the extent of gas accumulation and obstructing lesions in the urinary tract. It essentially plays a part in assessing the prognosis as well as in management decision making according to the classification mentioned above.

The pattern of gas distribution can be clearly observed on a CT scan. The pattern of gas can be 'Streaky' or 'Streaky and mottled' or 'Streaky and bubbly' among which the former two are more indicative of unfavorable outcome. Usually need regular follow up images as EPN is a dynamic condition.(Figure 1,Figure 2)

Gas collection in the perinephric area can be either rim like or crescent-shaped. Gas accumulation may also be seen in the renal vein, inferior vena cava, psoas muscle and perinephric areas.
USS-KUB reveals high echogenic areas with dirty shadowing and gas distribution over the region but its accuracy is around $69 \%$ and for plain radiographs its about $65 \%$.

Lab investigations usually reveal Leukocytosis, hyperglycemia, pyuria, elevated serum creatinine, high inflammatory markers, thrombocytopenia and positive blood cultures. Mostly the organisms found in blood culture coincides with the urine and the pus. E.coli would be the commonest organism followed by Klebsiella pneumoniae.

However, CT plays a vital role in diagnosis as well as planning the intensity of clinical care.

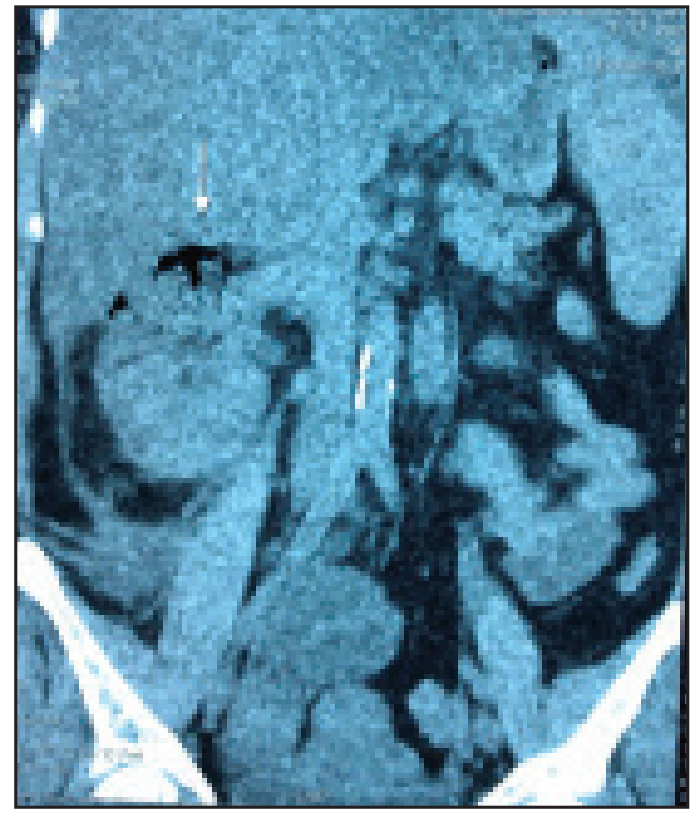

Figure 1: NCCT KUB of a poorly

controlled diabetic patient with EPN

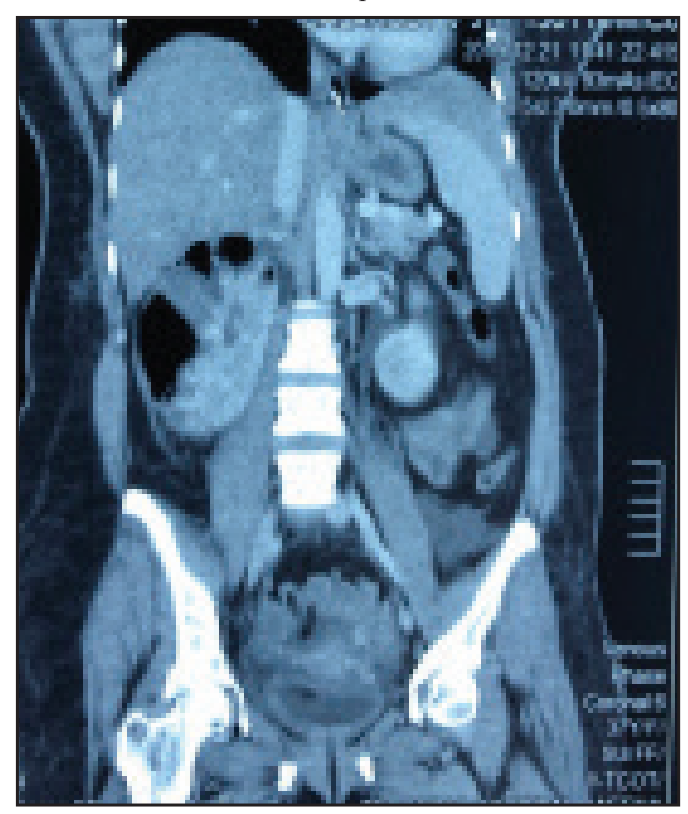

Figure 2: CT-IVU of same

patient next day(Worsening Type 1 EPN) 


\section{Management}

Since EPN is a severe life-threatening necrotizing infection of kidneys, early diagnosis and a proper management is mandatory to reduce mortality and morbidity.

Those who fail to respond to the standard treatment of pyelonephritis should be investigated with urgent CT scan of abdomen to confirm the diagnosis of EPN.

The treatment strategies include medical intensive care alone or PCD with medical intensive care or Medical intensive care with emergency nephrectomy. Emergency nephrectomy and/or open surgical drainage with antibiotic coverage was the accepted treatment option of EPN until the late 1980s. $(5,6) 40-50 \%$ of mortality rate is reported with this type management.

Later with advanced technology per cutaneous catheters developed as a desirable treatment option for EPN, which was first shown by Hudson et al.

Currently nephron sparing approach with PCD with or without elective nephrectomy at later stage is the main treatment of choice for EPN and the mortality rate is significantly low in this type of management (12).
Nephrectomy should be reserved for extensive form of EPN with multi organ failure or for patients who show a failed response to conservative management.

Conservative management is indicated in:

Class 1 and Class 2 EPN.

- $\quad$ Early cases associated with gas in the collecting system alone, with the patient otherwise stable.

Conservative management involves keeping the patient well hydrated and giving parenteral antibiotics. Control of blood glucose level is mandatory. In cases of urinary tract obstruction, percutaneous drainage or stent placement is indicated for relief of the obstruction.

Nephrectomy is indicated in:

1. Patients who fail to respond conservative management.

2. Situations where there is no access to $\mathrm{PCD} /$ stenting.

In addition to above management options, patients might need renal supportive care like dialysis or other modes of renal replacement therapy which seems to reduce the mortality rate significantly.

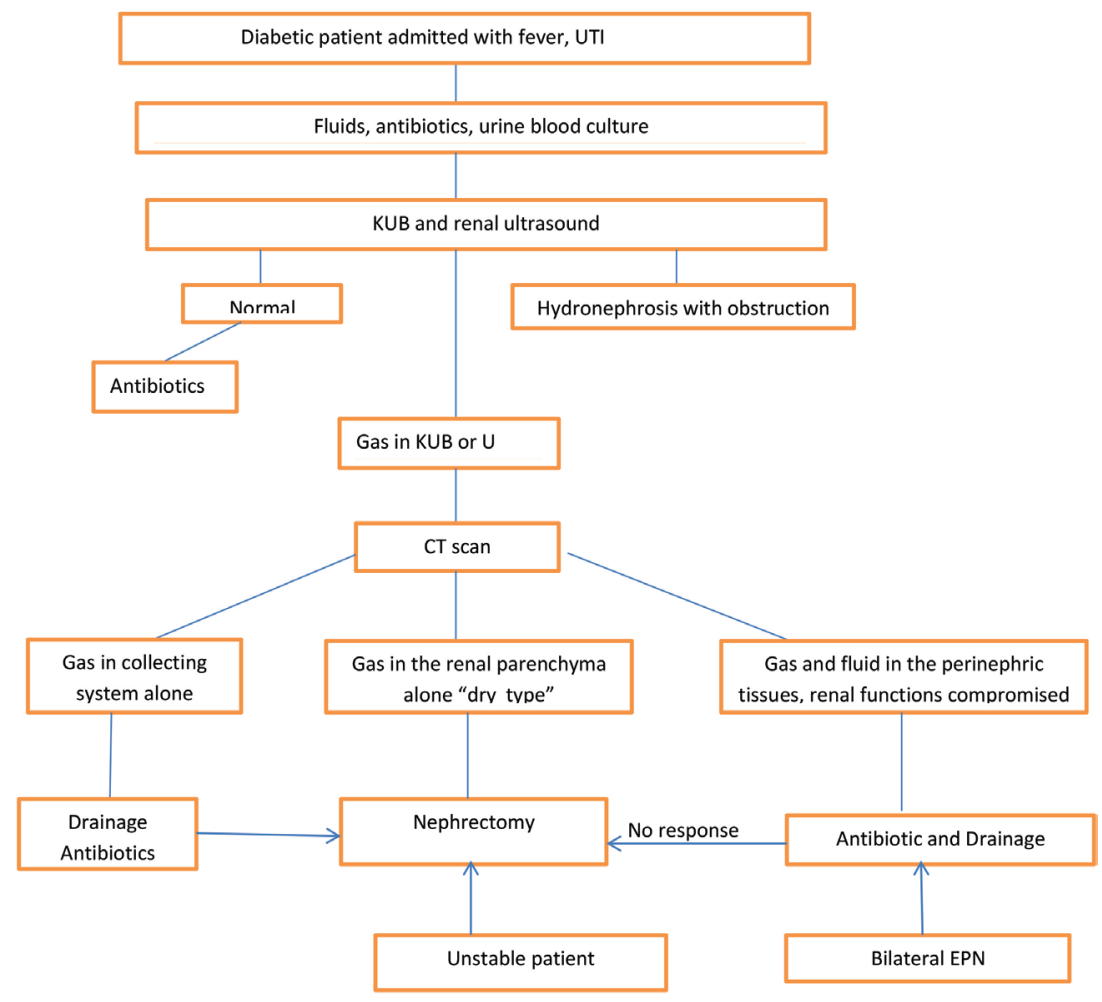

Figure 3: A diagram to describe presentation and management 


\section{References}

1. Ubee SS, McGlynn L, Fordham M. Emphysematous pyelonephritis. BJU Int. 2011;107(9):1474-1478. doi:10.1111/ j.1464-410X.2010.09660.x

2. Schicho A, Stroszczynski C, Wiggermann P. Emphysematous Cystitis: Mortality, Risk Factors, and Pathogens of a Rare Disease. Clin Pract. 2017;7(2):930. doi:10.4081/cp.2017.930.

3. Lu YC, Chiang BJ, Pong YH, et al. Emphysematous pyelonephritis: clinical characteristics and prognostic factors. Int J Urol. 2014;21(3):277-282. doi:10.1111/ iju.12244

4. Huang JJ, Tseng CC. Emphysematous pyelonephritis: clinicoradiological classification, management, prognosis, and pathogenesis. Arch Intern Med. 2000;160(6):797-805. doi:10.1001/ archinte.160.6.797

5. Chen MT, Huang CN, Chou YH, Huang $\mathrm{CH}$, Chiang CP, Liu GC. Percutaneous drainage in the treatment of emphysematous pyelonephritis: 10-year experience. JUrol. 1997;157(5):1569-1573.

6. Pontin AR, Barnes RD, Joffe J, Kahn D. Emphysematous pyelonephritis in diabetic patients. Br J Urol. 1995;75(1):71-74. doi:10.1111/j.1464-410x.1995.tb07237.x
7. Shokeir AA, El-Azab M, Mohsen T, ElDiasty T.Emphysematous pyelonephritis: a 15-year experience with 20 cases. Urology. 1997;49(3):343-346. doi:10.1016/S00904295(96)00501-8

8. Taussig AE. Pneumaturia, with report of a case. Boston Med Surg J 1907;156:769-74. DOI: 10.1056/NEJM190706131562403

9. Lu YC, Hong JH, Chiang BJ, et al. Recommended Initial Antimicrobial Therapy for Emphysematous Pyelonephritis: 51 Cases and 14-Year-Experience of a Tertiary Referral Center. Medicine (Baltimore). 2016;95(21):e3573. doi:10.1097/MD.0000000000003573

10. Schainuck LI, Fouty R, Cutler RE. Emphysematous pyelonephritis. A new case and review of previous observations. Am J Med. 1968 Jan. 44(1):134-9.1

11. Evanoff GV, Thompson CS, Foley R, Weinman EJ. Spectrum of gas within the kidney. Emphysematous pyelonephritis and emphysematous pyelitis. Am J Med. 1987;83(1):149-154. doi:10.1016/00029343(87)90511-0.

12. Somani BK, Nabi G, Thorpe P, et al. Is percutaneous drainage the new gold standard in the management of emphysematous pyelonephritis? Evidence from a systematic review. J Urol. 2008;179(5):1844-1849. doi:10.1016/j.juro.2008.01.019 